\title{
Nanoscale periodic domain patterns in tetragonal ferroelectrics: A phase-field study
}

\author{
A. Renuka Balakrishna, ${ }^{1, *}$ J. E. Huber, ${ }^{1}$ and I. Münch ${ }^{2}$ \\ ${ }^{1}$ Department of Engineering Science, University of Oxford, Parks Road, Oxford OX1 3PJ, England, United Kingdom \\ ${ }^{2}$ Institute for Structural Analysis, Karlsruhe Institute of Technology, Kaiserstraße 12, 76131 Karlsruhe, Germany
}

(Received 5 November 2015; revised manuscript received 18 March 2016; published 31 May 2016)

\begin{abstract}
Ferroelectrics form domain patterns that minimize their energy subject to imposed boundary conditions. In a linear, constrained theory, that neglects domain-wall energy, periodic domain patterns in the form of multirank laminates can be identified as minimum-energy states. However, when these laminates (formed in a macroscopic crystal) comprise domains that are a few nanometers in size, the domain-wall energy becomes significant, and the behavior of laminate patterns at this scale is not known. Here, a phase-field model, which accounts for gradient energy and strain energy contributions, is employed to explore the stability and evolution of the nanoscale multirank laminates. The stress, electric field, and domain-wall energies in the laminates are computed. The effect of scaling is also discussed. In the absence of external loading, stripe domain patterns are found to be lower-energy states than the more complex, multirank laminates, which mostly collapse into simpler patterns. However, complex laminates can be stabilized by imposing external loads such as electric field, average strain, and polarization. The study provides insight into the domain patterns that may form on a macroscopic single crystal but comprising nanoscale periodic patterns, and on the effect of external loads on these patterns.
\end{abstract}

DOI: 10.1103/PhysRevB.93.174120

\section{INTRODUCTION}

Domains are regions of a ferroelectric crystal with uniform polarization. The arrangement, or pattern, of these domains is of great importance in determining the properties of a ferroelectric at the nanoscale [1-7]. For example, the effective piezoelectric coefficients depend upon the fractions of different domain types present [6], and enhanced actuation can be achieved by using specific domain patterns [7]. Engineered configurations of domains have been used to make improved actuators, sensors, and energy harvesting devices [8]. Domain patterns are particularly important to the working of nanoscale devices, where relatively few domains are present [9-12].

What determines the pattern of domains? Where any two domains meet, a domain wall forms, and this has surface energy proportional to the wall area. But there is also potential energy, proportional to domain volume, associated with externally applied stresses and electric fields. Minimization of energy then produces a competition between the individual energy contributions, leading to specific patterns. In the absence of external loads, a single domain state minimizes energy; likewise, for boundary conditions consistent with a uniform stress or electric field, the single domain state is an energy minimizer. However, boundary conditions that impose an average strain or electric displacement can lead to a mixture of domains. Once a mixture of domains becomes favorable, energy minimization produces arrangements of domains that relieve internal stresses and electric fields. Thus "head-to-tail" polarization arrangements [13-15] form across domain walls, and the spontaneous strains in adjacent domains satisfy compatibility conditions, well known from the crystallographic theory of martensite $[16,17]$. Where these conditions cannot be met, the resulting misfit strains cause internal stresses and incompatible polarization causes electric

*Corresponding author: ananya.renukabalakrishna@eng.ox.ac.uk. fields; both produce a contribution to the net energy. The compatibility rules give a "constrained theory" that has been widely used to study pattern formation in ferroelectrics and other materials $[18,19]$. Here, we use this approach to provide a starting point for our simulations.

The fact that the domain-wall energy scales with area, while the potential energy scales with volume, introduces an intrinsic, material specific length scale. Thus energy minimization can determine both the pattern of domains and the scale at which this pattern forms. At a sufficiently coarse scale, the domain-wall energy becomes negligible, and a constrained theory, based on compatibility alone, is sufficient. However, for the prediction of fine domain patterns, the contribution of domain-wall energy becomes significant. The key length scale for typical ferroelectrics is of the order of nanometers, comparable with the finest spacing of observed domains [20-22].

Of course, the materials may not behave as perfect minimizers of energy. Kinetics has a central role in determining the ways that domains can evolve. For example, the multiwell nature of the energy dictates that even if the system is driven towards minimum energy it may only reach a local energy minimum. Defects, such as trapped charge [23], dislocations [24], dopant atoms [25], and so forth, also play a role, affecting both the energetics and kinetics [26]. Nevertheless, substantial progress in understanding domain patterns has been made by considering energy minimization in perfect crystals.

Domain patterns in single-crystal ferroelectrics commonly take the form of nearly periodic laminations [1,15,27-31]. We can define a rank-1 laminate, as a pattern comprising alternating layers of two domain types, each of which is a single-crystal variant. Examples include alternating bands of 180 or $90^{\circ}$ domains. Similarly, simple laminations can themselves be layered together to produce a higher rank laminate. Based on the linear constrained theory of laminates, Tsou et al. [18] identified several rank-2 periodic domain patterns in tetragonal ferroelectrics. Of these, a layered stripe domain pattern and herringbone domain pattern are both well 
known and commonly observed [13,14,30,32]. Meanwhile, certain other patterns are rarely seen in experiments [33,34], though elements of these patterns sometimes appear [15]. In the present work, we consider periodic polarization patterns formed in macroscopic single crystals, but with nanoscale domains. These polarization patterns are referred to as nanoscale periodic patterns. The theory employed by Tsou et al. [18] neglected gradient energy due to domain walls and the elastic and dielectric energy due to disclinations generated at the junctions of domains. However, these factors could be expected to be of importance, especially in nanoscale periodic patterns $[35,36]$. This leads us to consider the following questions: Among the periodic laminates identified by the constrained theory, which are stable when gradient and elastic energy are considered? Which patterns constitute global energy minima, and does this depend on the scale? Can imposed states of average strain or polarization stabilize specific patterns, allowing the nanoengineering of domain patterns?

In the present work, we use a phase-field approach based on the time-dependent Ginzburg-Landau equations [37-41]. This modeling approach is well established for ferroelectrics and has been successfully applied to study defects [42,43], domain structures in thin films [44,45], the effect of flexoelectric coupling on domain patterns [46-54], and microstructural evolution under electromechanical loading [55]. The phasefield approach has made a significant contribution in understanding nanoscale ferroelectrics and reliably accounts for energy contributions from polarization gradients and misfit strains at domain walls [56]. Several reviews provide a broader appreciation of the capabilities of the method $[45,57,58]$. The phase-field simulations have also been employed to simulate macroscopic ferroelectric properties by modeling periodic boundary conditions [1,38,59-64]. For example, systematic studies on the effect of electromechanical boundary conditions on hysteresis, butterfly loops, and polarization switching have been conducted using periodic conditions [38,59-63]. While there exists a substantial literature making use of phase-field models to study periodic patterns with stripelike features in both two-dimensional (2D) [38,59-62] and three-dimensional (3D) $[63,65]$ simulations, to date there is relatively little work done to systematically explore the stability of nanoscale periodic patterns with 2D checkerboardlike features or with cylindrical domains in three dimensions that are predicted to form in tetragonal ferroelectrics $[27,28,66]$, which is the goal of the present work. In this paper, the stability of these complex nanoscale periodic patterns under externally applied strain and electric field is also explored.

The phase-field model $[42,67]$ used in the present work enables an initial assessment of the stability of rank-1 and rank-2 nanoscale periodic domain patterns in tetragonal ferroelectrics. We then examine the internal fields in the domain patterns and consider the effect of scaling on internal energy. Several of the rank-2 periodic patterns when modeled with nanoscale domains collapse into simpler states. However, they can be stabilized by external loads, and examples of this are given. We also show that, with suitable loading, simple rank-1 domain patterns can be made to evolve into more complex, rank-2 patterns, suggesting a mechanism for the formation of complex domain patterns. Throughout, the phase-field results

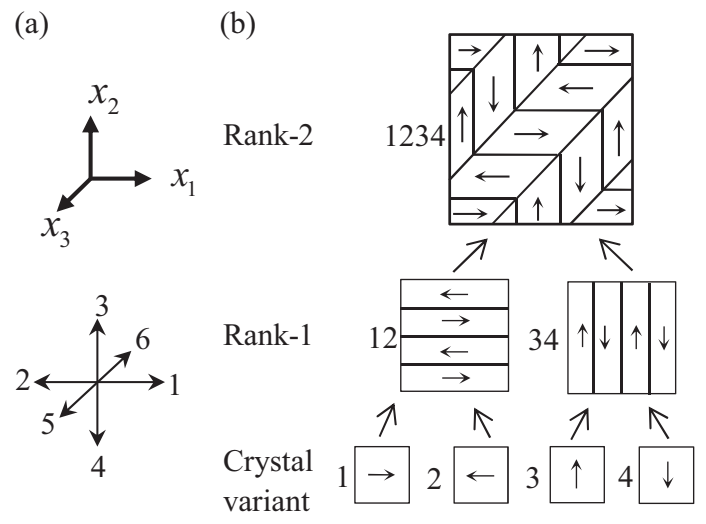

FIG. 1. (a) Coordinate axes and polarization directions of the six crystal variants in the tetragonal system. (b) A rank-2 tree diagram illustrating the domain pattern "1234".

are compared with the existing literature on periodic domain patterns.

\section{PROBLEM DESCRIPTION AND MODEL}

The objective is to test the stability of nanoscale periodic patterns that form in a macroscopic single crystal. To do this, a set of rank-1 and rank-2 periodic laminates identified by Tsou et al. [18] is modeled using phase-field methods. Following Tsou et al. $[18,66]$, the multirank laminates are labeled on the basis of the crystal variants present in the domain pattern. There are six crystal variants in the polar tetragonal system, with distinct spontaneous polarization states as shown in Fig. 1(a). The structure of a multirank laminate consists of layers which are themselves subdivided into finer layers, the finest laminations comprising material of a single-crystal variant. The arrangement of layers is readily described using a hierarchical tree structure [68] [see Fig. 1(b)]. At the lowest level are single-crystal variants, numbered according to their polarization orientation. Ascending the tree diagram, higher rank laminates are labeled so as to show the crystal variants present, reading from left to right across the lowest level of the tree. Thus, in Fig. 1(b), the rank-1 domain patterns are labeled as "12" and "34." These rank-1 domain patterns can be laminated together to form a rank-2 herringbone domain pattern, labeled "1234."

There are many laminate patterns that can be formed in this way. However, most are reflections, rotations, or inversions of other patterns. Taking out these symmetrical copies, Tsou et al. [18] found just two distinct laminates of rank-1 and eight of rank-2. We model nanoscale periodic cells of these patterns and seek equilibrium states.

A phase-field model developed by Landis and coworkers $[42,67]$ is used. This model has been calibrated using the material properties of barium titanate $\left(\mathrm{BaTiO}_{3}\right)$ in its tetragonal phase [67]. The order parameter is the local polarization $P_{i}$, and the polarization field is allowed to approach equilibrium by relaxation using the Ginzburg-Landau equation [67]:

$$
\left(\frac{\partial \psi}{\partial P_{i, j}}\right)_{, j}-\frac{\partial \psi}{\partial P_{i}}=\beta \dot{P}_{i}
$$


Here $\psi$ is the Helmholtz free energy per unit volume. An arbitrary polarization viscosity, $\beta$, is introduced for numerical purposes, and is controlled as a relaxation parameter. Equilibrium states satisfy Eq. (1) with $\beta=0$. The Helmholtz free energy is described by

$$
\begin{aligned}
\psi= & \frac{1}{2} a_{i j k l} P_{i, j} P_{k, l}+\frac{1}{2} \bar{a}_{i j} P_{i} P_{j}+\frac{1}{4} \overline{\bar{a}}_{i j k l} P_{i} P_{j} P_{k} P_{l} \\
& +\frac{1}{6} \overline{\overline{\bar{a}}}_{i j k l m n} P_{i} P_{j} P_{k} P_{l} P_{m} P_{n} \\
& +\frac{1}{8} \overline{\overline{\bar{a}}}_{i j k l m n r s} P_{i} P_{j} P_{k} P_{l} P_{m} P_{n} P_{r} P_{s} \\
& +b_{i j k l} \varepsilon_{i j} P_{k} P_{l}+\frac{1}{2} c_{i j k l} \varepsilon_{i j} \varepsilon_{k l}+\frac{1}{2} f_{i j k l m n} \varepsilon_{i j} \varepsilon_{k l} P_{m} P_{n} \\
& +\frac{1}{2} g_{i j k l m n} \varepsilon_{i j} P_{k} P_{l} P_{m} P_{n}+\frac{1}{2 \kappa_{0}}\left(D_{i}-P_{i}\right)\left(D_{i}-P_{i}\right)
\end{aligned}
$$

where $D_{i}$ is the electric displacement, $\varepsilon_{i j}$ is the strain tensor, and $\kappa_{0}$ is the permittivity of free space. The tensorial coefficients $\mathbf{a}, \mathbf{b}, \mathbf{c}, \mathbf{f}$, and $\mathbf{g}$ are given, along with further details of the model and the material properties of $\mathrm{BaTiO}_{3}$ at room temperature $\left(22^{\circ} \mathrm{C}\right)$ in the work by Landis and coworkers $[42,67]$. These parameters were chosen to represent the multiwell free energy and provide the correct symmetries to model the material properties in the tetragonal phase. In Eq. (2), the energy contribution from surface energy is assumed to be negligible since the work focuses on macroscopic crystals. The phase-field problem is solved using finite element methods, with eight-noded quadratic elements in 2D simulations or brick elements in 3D simulations. An element size of $1 \mathrm{~nm}$ was chosen, such that domain walls typically span over about three elements.

The material model is fully 3D, but several of the domain patterns considered have polarization in a single plane with all domain walls perpendicular to that plane. In these cases the prismatic nature of the patterns is exploited, allowing them to be modeled in two dimensions, with plane strain and electricfield conditions. This is practically expedient because the simulations are computationally intensive. However, it does limit the simulation by preventing out-of-plane polarization. Our experience with a wider range of simulations indicates that this restriction does not greatly affect the model outcomes. Domain patterns with out-of-plane polarizations are modeled in three dimensions. To model a regular repeating laminate structure, periodic boundary conditions are imposed (see Fig. 2). A periodic square of side $\mathrm{L}=40 \mathrm{~nm}$, Fig. 2(a), and a periodic cuboid with depth $D<L$, Fig. 2(b), are modeled in the 2D and 3D simulations, respectively. These periodic cells when repeated infinitely form a macroscopic object like a thin film or a single crystal.

The midelements of the periodic cell shown in Figs. 2(a) and 2(b) have simple supports, while periodic conditions are enforced on the boundary nodes, controlling the displacement $u_{i}$, electric potential $\phi$, and polarization $P_{i}$. For a typical node $R$, these conditions are

$$
\begin{aligned}
\left.u_{i}\left(L, x_{2}, x_{3}\right)\right|_{R}-\left.u_{i}(L, 0,0)\right|_{\mathrm{B}} & =\left.u_{i}\left(0, x_{2}, x_{3}\right)\right|_{R_{m}}-\left.u_{i}(0,0,0)\right|_{\mathrm{A}}, \\
\left.\phi\left(L, x_{2}, x_{3}\right)\right|_{R} & =\left.\phi\left(0, x_{2}, x_{3}\right)\right|_{R_{m}}, \\
\left.P_{i}\left(L, x_{2}, x_{3}\right)\right|_{R} & =\left.P_{i}\left(0, x_{2}, x_{3}\right)\right|_{R_{m}} \quad i=1, n .
\end{aligned}
$$

(a)

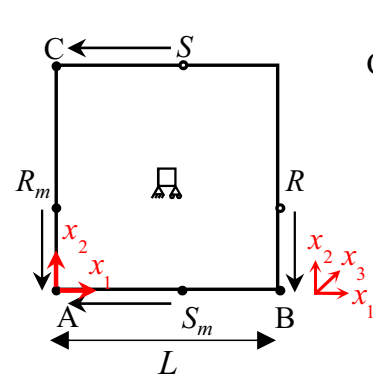

(b)

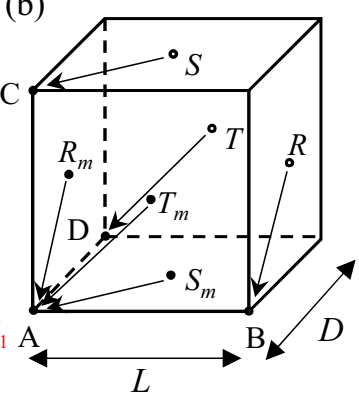

FIG. 2. Schematic representation of the periodic cell and boundary conditions on (a) a square of side $L$ and (b) a cuboid of depth $D$. Arrows indicate the nodal relations in periodic boundary conditions.

Here $n$ is the number of dimensions, and for 2D simulations $x_{3}=0$. The notation $\left.\right|_{X}$ is used to indicate a value at a node $X$ as labeled in Fig. 2. Similar conditions apply to typical node $S$ and (in the 3D case) $T$. Care is needed at corners in two dimensions and at edges in three dimensions to avoid duplication of boundary conditions. These boundary conditions allow the domain pattern to adopt periodic strain fields $\varepsilon_{i j}\left(x_{1}, x_{2}, x_{3}\right)$ with zero average stress. That is, the strain fields $\varepsilon_{i j}$ are dependent on the relative displacement between the corner nodes, for example, node $\mathrm{A}$ and node $\mathrm{B}$, which are not bound. These corner nodes are free to adopt any displacement values $u_{i}$ such that the periodic cell possesses zero average stress. Meanwhile, periodic values of electric potential $\phi$ with zero average electric field and polarization $P_{i}$ are imposed on the boundary nodes. We shall later refer to the boundary conditions represented by Eq. (3) as "zero external load" conditions. Equation (3) imposes the boundary conditions of a bulk material and previous literature $[3,14,15,30,32,69-71]$ indicates that periodic domain patterns have been experimentally observed in bulk samples.

The phase-field simulation is initialized by imposing a polarization field consistent with the repeating unit of a laminate pattern on the periodic cell. The nodal polarization values are set equal to the spontaneous polarization of the domain, producing sharp discontinuities at domain walls in the initial state. The displacement and electric potential values are initialized at zero. Note that this initial state may not satisfy electromechanical compatibility conditions, however it provides a state of the order parameter $P_{i}$ that approximates an equilibrium pattern.

During the early stages of each simulation, high values of polarization viscosity, $\beta$, are used to facilitate the resolution of domain walls into a continuous polarization field and displacement field. The sharp discontinuity at the domain walls rapidly relaxes, but the overall pattern of polarization is otherwise almost unchanged at this stage. Once this initial settling has occurred-a condition that will be referred to as the "settled state"- the free energy of the pattern, though not necessarily yet in equilibrium, is calculated. Subsequently, larger relaxation steps are used, allowing the simulation to evolve towards equilibrium, with the possibility of changing the polarization pattern in the process. 


\section{RESULTS AND DISCUSSION}

Before presenting the results of the simulations, several normalizations are introduced. The Helmholtz free energy per unit volume is normalized as $\tilde{\psi}=\left(\psi_{0}-\psi\right) / \psi_{0}$, where $\psi_{0}$ corresponds to the energy per unit volume of a spontaneously polarized monodomain. Here $\psi_{0}<0$, while $\psi=0$ corresponds to the cubic state with $P_{i}=0$. The polarization and strains are normalized by $P_{0}=0.26 \mathrm{C} / \mathrm{m}^{2}$ and $\varepsilon_{0}=0.0082$, respectively, which arises from the spontaneous state of barium titanate. Note that $\mathrm{BaTiO}_{3}$ has tetragonal symmetry and possesses a transverse spontaneous strain $\varepsilon_{0}^{\mathrm{t}}=-0.0027$. $E_{0}=21.82 \mathrm{MV} / \mathrm{m}$ is the normalization constant for electric field and corresponds to the critical field required to cause homogeneous $180^{\circ}$ switching of a spontaneously polarized monodomain. Equating mechanical and electrical energies, the normalization for stress is derived as $\sigma_{0}=E_{0} P_{0} / \varepsilon_{0}=$ $692 \mathrm{MPa}$. The characteristic length scale in this model is $l_{0}=\sqrt{a_{0} P_{0} / E_{0}}=1 \mathrm{~nm}$, where $a_{0}=1 \times 10^{-10} \mathrm{Vm}^{3} / \mathrm{C}$ is a coefficient used in specifying the gradient energy term in Eq. (2) [67]. The electric potential normalization is derived from the electric field and length scale normalizations as $\phi_{0}=E_{0} l_{0}=0.022 \mathrm{~V}$.

\section{A. Rank-1 domain patterns}

At first, we test the stability of the rank-1 domain patterns under zero external load conditions as given by Eq. (3). The two rank-1 domain patterns identified in tetragonal ferroelectrics are the " 12 " laminate pattern with alternating $180^{\circ}$ domain bands and the "14" laminate pattern with alternating $90^{\circ}$ domain stripes (see Fig. 3). The "12" domain pattern is modeled with two different domain spacings $s=20$ and $10 \mathrm{~nm}$ defined along cross section AA [see Figs. 3(a) and 3(b)]. Similarly the "14" domain pattern is modeled at two spacings, with $s=14.1$ and $7.1 \mathrm{~nm}$ defined along cross section BB, as shown in Figs. 3(c) and 3(d).
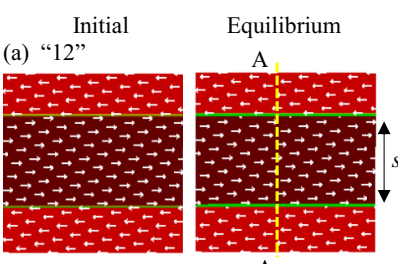

A
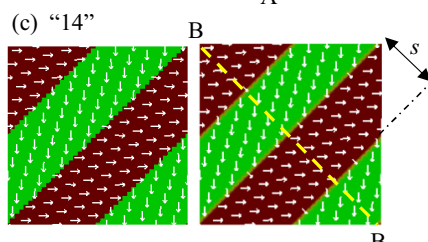

$\stackrel{x_{2}}{\longrightarrow} x_{1}$
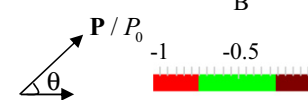

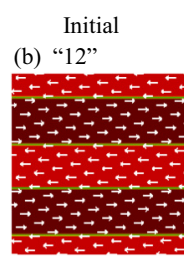

(d) " 14 "

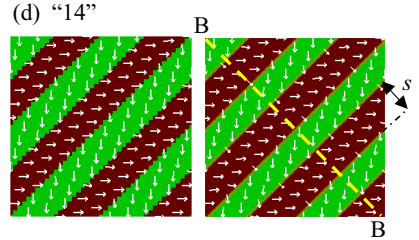

FIG. 3. Testing the stability of rank-1 domain patterns under zero external load conditions. $\mathbf{P} / P_{0}$ values in (a) laminate " 12 " with $s=$ $20 \mathrm{~nm}$, (b) laminate " 12 " with $s=10 \mathrm{~nm}$, (c) laminate " 14 " with $s=14.1 \mathrm{~nm}$, and (d) laminate " 14 " with $s=7.1 \mathrm{~nm}$. AA and BB indicate cross sections perpendicular to the domain walls. (a)

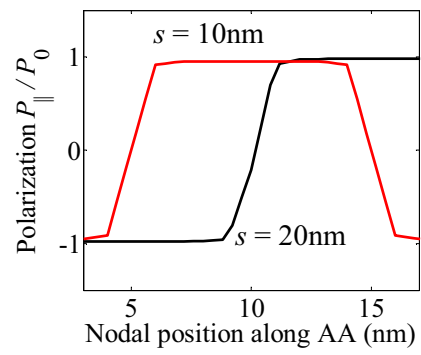

(b)

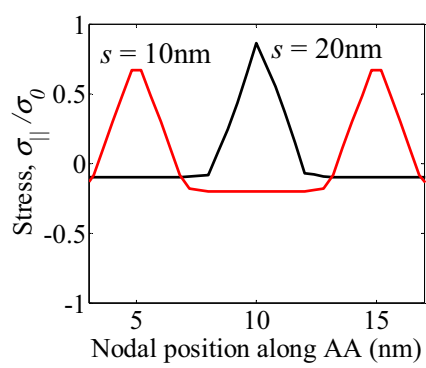

FIG. 4. Nodal values of (a) polarization $P_{\|}$, parallel to the domain wall, and (b) stress component $\sigma_{\|}$, parallel to the domain wall on cross section $\mathrm{AA}$ in the $180^{\circ}$ band domain pattern.

At equilibrium, the sharp domain walls in the initial states become smooth, but the patterns are otherwise unchanged [see Figs. 3(a)-3(d)]. Nanoscale periodic patterns with bundles of stripelike or bandlike features have been observed to be stable in experiments on $\mathrm{BaTiO}_{3}$ [27] for example by McGilly et al. [15] and Schilling et al. [29,70]. This stability of nanoscale patterns with 180 or $90^{\circ}$ domains is in agreement with the phase-field simulation results [see Figs. 3(a)-3(d)].

The variations in polarization and stress developed on section AA in the $180^{\circ}$ domain pattern "12" are shown in Figs. 4(a) and 4(b). From Fig. 4(a), the domain-wall width is close to $2 \mathrm{~nm}$, consistent with the value for an isolated domain wall given by Su and Landis [67], and is unaffected by changing the domain spacing from 10 to $20 \mathrm{~nm}$. These values are also consistent with the results from the quasi-one-dimensional analysis of tetragonal twins by Cao and Cross [72] and match separate calculations by Hlinka and Márton [73]. Away from the domain walls, the polarization magnitude is close to the spontaneous value (within 1\%) and there is no polarization perpendicular to the walls. The electric field is everywhere negligible. The domain-wall width in ferroelectrics can also be influenced by surface effects and elastic interaction between nonferroelastic domain walls, which are discussed in previous investigations $[74,75]$.

At equilibrium, stresses develop parallel to the $180^{\circ}$ walls as shown in Fig. 4(b), while stresses perpendicular to the walls are zero. Here, these stresses correspond to the values of $\sigma_{11}$ and $\sigma_{22}$, respectively. With spacing $s=20 \mathrm{~nm}$ the tensile stress peaks at about $0.85 \sigma_{0}$ in the domain walls, balanced by compressive stresses of about $-0.1 \sigma_{0}$ in the domains. On decreasing the spacing to $s=10 \mathrm{~nm}$, the peak stress reduces to about $0.65 \sigma_{0}$, while the compressive stresses in the domains double in magnitude, becoming $-0.2 \sigma_{0}$. The energy per unit area associated with the presence of domain walls was calculated using

$$
\gamma_{\mathrm{w}}=\frac{\int_{V}\left(\psi-\psi_{0}\right) d V}{A_{\mathrm{w}}}
$$

where $A_{\mathrm{w}}$ is the wall area within the periodic cell of volume $V$. We found $\gamma_{\mathrm{w}} \sim 13.1 \mathrm{~mJ} / \mathrm{m}^{2}$ when $s=20 \mathrm{~nm}$ and $\gamma_{\mathrm{w}} \sim$ $12.6 \mathrm{~mJ} / \mathrm{m}^{2}$ for $s=10 \mathrm{~nm}$. This compares with the value of $14.8 \mathrm{~mJ} / \mathrm{m}^{2}$ for the case of an isolated domain wall $s \rightarrow \infty$ [67]. It is interesting to note that the domain-wall energy in the periodic laminate is less than the energy of an isolated domain 
(a)

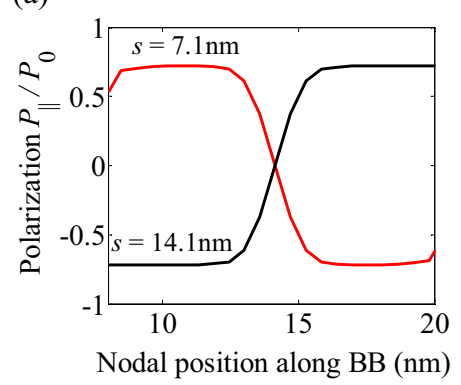

(b)

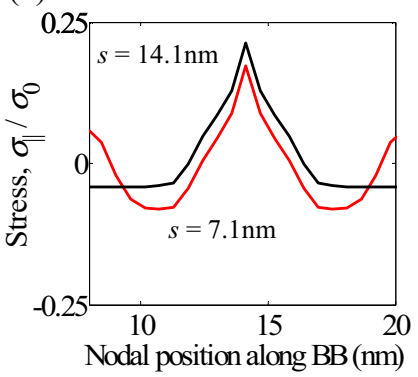

(c)

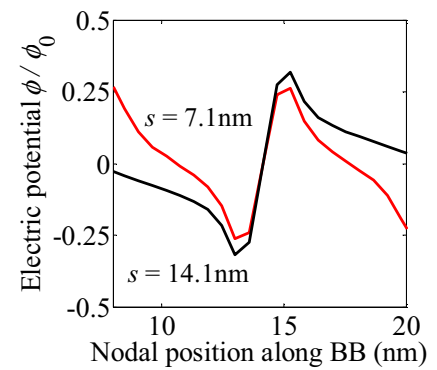

FIG. 5. Nodal values of (a) polarization $P_{\|}$, parallel to the domain wall, and (b) stress component $\sigma_{\|}$and (c) electric potential $\phi$ on cross section $\mathrm{BB}$ in the $90^{\circ}$ stripe domain pattern.

wall, and dependent upon the domain spacing. The decrease in stresses at the domain walls accounts for the difference. Similar magnitudes of domain-wall energy have been found in previous investigations [76].

The polarization variation on section $\mathrm{BB}$ in the $90^{\circ}$ stripe domain pattern "14," with spacing of 14.1 and $7.1 \mathrm{~nm}$, is shown in Fig. 5(a). Away from the domain walls, the polarization magnitude approaches $P_{0}$ with components of magnitude $P_{0} / \sqrt{2}$ parallel and perpendicular to the domain walls. From Fig. 5(a) the $90^{\circ}$ domain-wall width is $\sim 3 \mathrm{~nm}$, in agreement with $\mathrm{Su}$ and Landis [67].

At equilibrium, tensile stresses parallel to the $90^{\circ}$ domain walls develop, balanced by compressive stresses in the domains [see Fig. 5(b)]. The stresses at the domain walls peak at about $0.2 \sigma_{0}$ for $s=14.1 \mathrm{~nm}$ and reduce to $0.17 \sigma_{0}$ when $s=7.1 \mathrm{~nm}$. This differs from the value of $\sim 0.33 \sigma_{0}$ calculated for the case $s \rightarrow \infty$ [67]; the difference can be attributed to the fine spacing of the domains, which places the domains into significant compression and hence affects the stress distribution in the domain walls. The domain-wall energies are calculated to be $\sim 6.0 \mathrm{~mJ} / \mathrm{m}^{2}$ for $s=14.1 \mathrm{~nm}$ and $\sim 5.8 \mathrm{~mJ} / \mathrm{m}^{2}$ for $s=7.1 \mathrm{~nm}$. These values are consistent with the energy calculations by $\mathrm{Su}$ and Landis [67]. The stresses developed in the $90^{\circ}$ stripe domain pattern are rather low in comparison to the $180^{\circ}$ band domain pattern, and this accounts for the difference in the 90 and $180^{\circ}$ domain-wall energies.

Nonzero local electric fields are developed at the $90^{\circ}$ domain walls, as shown by the change in electric potential values in Fig. 5(c). Note that the pattern has a nonzero average polarization. The electric fields are in the direction opposite to this net polarization and cause deviation from the spontaneously polarized state at the walls. A consequence is that there are electric fields in the domains that are aligned with the average polarization. With $s=14.1 \mathrm{~nm}$ the change in electric potential across a domain wall is about $\Delta \phi \sim 0.6 \phi_{0}$, corresponding to an electric field of magnitude $\sim 0.2 E_{0}$. On decreasing the spacing to $s=7.1 \mathrm{~nm}, \Delta \phi$ drops to $\sim 0.5 \phi_{0}$ with the corresponding electric-field magnitude reduced to $\sim 0.17 E_{0}$. However, the electric field in the polarized domains increases with decrease in spacing.

We also tested whether keeping the cell size the same but changing the relative position of the domain walls affects the overall energy. If moving one domain wall within the cell affects the energy then there could be a minimum-energy arrangement—effectively a "preferred" separation distance between domain walls. We found that this is not the case: the domain walls in the cell were in neutral equilibrium over a wide range of separation distances. Details of these simulations are omitted for brevity. The finding is consistent with observations that commonly show an approximately periodic pattern, but with considerable variation in distances between domain walls [14,34]. This is significant because it indicates that perfectly engineered domain configurations with regular spacing are unlikely to form naturally and will require special conditions to enforce regularity. An example of such conditions is given later in Sec. III C.

\section{B. Rank-2 domain patterns}

Next we test the stability of rank-2 periodic domain patterns under the zero external load conditions. Among the rank-2 laminates identified by Tsou et al. [18] four domain patterns which contain polarization only in a single plane with domain walls perpendicular to that plane are first tested [see Figs. 6(a)-6(d)]. All of the rank-2 domain patterns shown in Figs. 6(a)-6(d) are modeled with zero average stress and electric-field conditions as described by Eq. (3). Here, note that the strain fields $\varepsilon_{i j}$ adopted by the periodic cell vary based on the type of domain pattern imposed in the initial state. The volume average free energy $\tilde{\psi}$, average axial components of strain $\tilde{\varepsilon}_{i j}$, and polarization $\tilde{P}_{i}$, adopted by these domain patterns in their settled states, are given in Table I. Each pattern was simulated in a square region of side $L=40 \mathrm{~nm}$ using plane strain and electric-field conditions.

The herringbone domain pattern "1234" shown in Fig. 6(a) is found to be stable at this scale. This domain pattern contains several domain walls in the periodic cell, which accounts for its relatively high free energy in comparison to the rank-1 domain patterns.

Stresses in the herringbone domain pattern are developed at the $180^{\circ}$ domain walls, while nonzero local electric fields are observed at the $90^{\circ}$ domain walls. During relaxation the domain walls drifted slightly, resulting in a small magnitude of net polarization $\left(\sim 0.05 P_{0}\right)$ due to the slightly unequal sizes of the $\pm P_{2}$ domains at equilibrium. Herringbone patterns are observed to be stable in ferroelectrics [14,27,30,77], thereby confirming the phase-field results in Fig. 6(a).

The periodic domain pattern in Fig. 6(b) shows laminate " 1323 " which contains alternating bands of $\pm P_{1} 180^{\circ}$ domains 

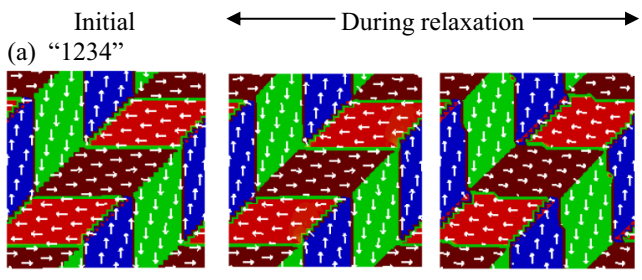

Equilibrium

(b) " 1323 "
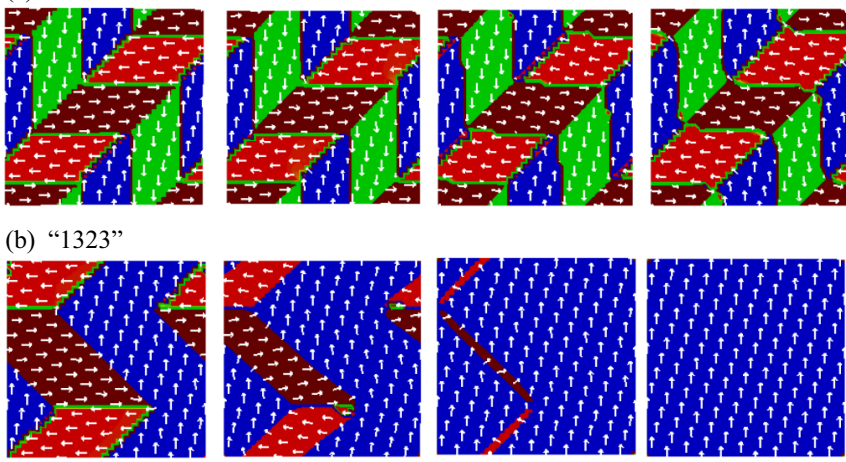

(c) "1434"
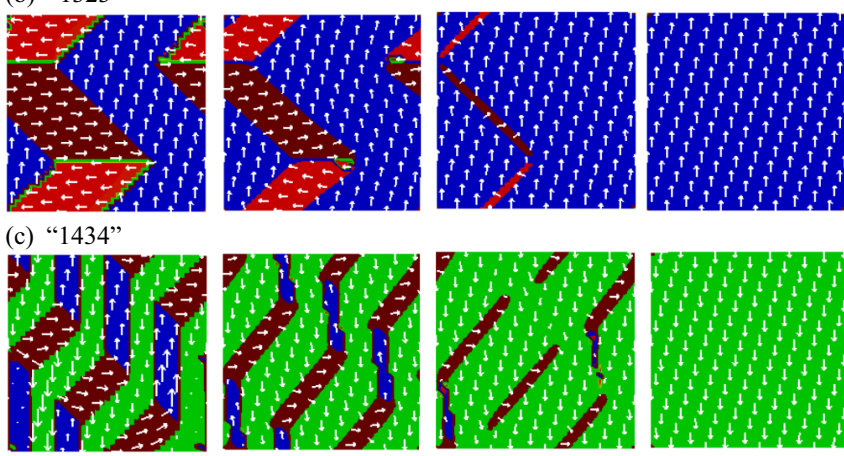

(d) " 1324 "
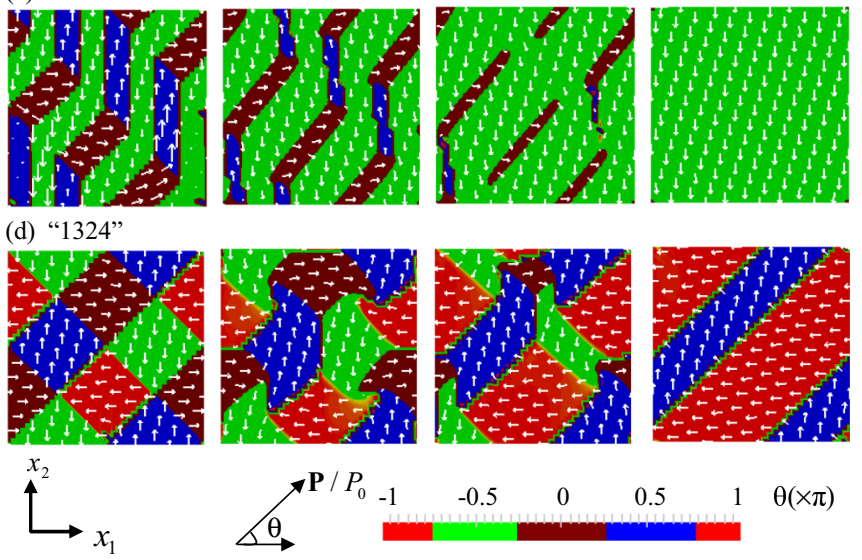

FIG. 6. Testing the stability of rank-2 domain patterns with inplane polarizations under zero external load conditions, laminates (a) "1234," (b) “1323," (c) “1434," and (d) “1324.” Subfigures map domain rearrangements during relaxation from the initial state (far left) to the final equilibrium state (far right).

interspersed with a zig-zag stripe which is a single $+P_{2}$ domain. This domain pattern was identified as a minimumenergy state in the linear constrained theory [18]; that is, all domain walls satisfy compatibility conditions. However, when "1323" is modeled with nanoscale domains, the pattern is found to be unstable: during relaxation it dissolves to form a uniformly polarized $+P_{2}$ domain. At the settled state, i.e., where domain walls are resolved but the polarization pattern is unchanged, the $\pm P_{1}$ domains experience a tensile stress $\sigma_{22}$, while the $P_{2}$ domains experience compressive stress $\sigma_{22}$. This is to be expected because the spontaneous strains component
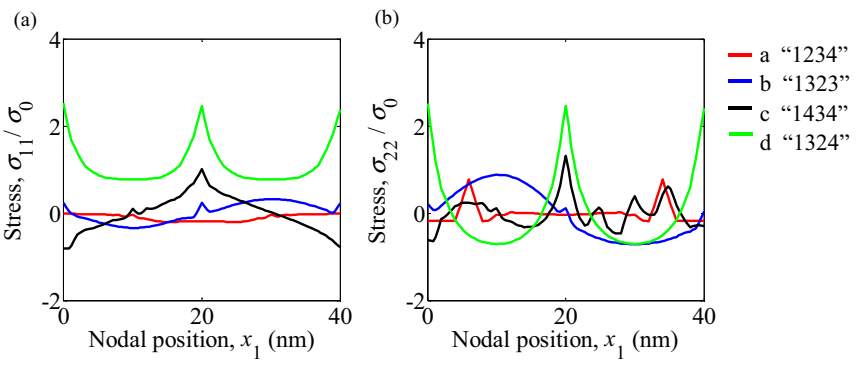

FIG. 7. Stress distributions in the rank-2 periodic domain patterns with in-plane polarizations: (a) $\sigma_{11}$ and (b) $\sigma_{22}$ at a cross section $x_{2}=L / 2$, at varying $x_{1}$ positions.

$\varepsilon_{22}$ differs between the $\pm P_{1}$ domains (where it is $\varepsilon_{0}^{\mathrm{t}}$ ) and the $P_{2}$ domains (where it is $\varepsilon_{0}$ ). Since these vertical zig-zag stripes are assumed to be perfectly adhered to each other, misfit stress arises from the difference in spontaneous strain. Figure 7 shows the direct stresses $\sigma_{11}, \sigma_{22}$ evaluated at a cross section of the model where $x_{2}=L / 2$. It is clear that the herringbone pattern (label "a") has relatively low values of stress throughout, while the tensile and compressive stresses in the alternating bands of the "1323" domain pattern (label "b") are of order $\sigma_{0}$. This contribution of elastic energy due to misfit stress explains the lack of stability and accounts for the net energy of the domain pattern (see Table I).

An additional factor contributing to the high energy of the "1323" domain pattern is the presence of disclinations at the junctions of domains. These arise because the unstressed condition of a $90^{\circ}$ domain wall produces a slight rotation of the crystal lattice, dependent on the tetragonal $c: a$ ratio. In barium titanate this rotation is about $0.63^{\circ}$. Upon circulating a continuous junction of domains, no net rotation of the crystal lattice is permissible. Consequently the domains are stressed if the set of domain walls at a junction would otherwise produce a net rotation. For example, the domains in the "1234" herringbone pattern are so arranged that there is no net lattice rotation upon circulating any junction: opposite rotations on passing $90^{\circ}$ domain walls cancel. However, in the " 1323 " pattern, circulation of a domain junction implies two consecutive lattice rotations of $0.63^{\circ}$, resulting in a disclination.

The domain rearrangement process during relaxation in Fig. 6(b) indicates the growth of domains with a larger initial volume fraction, i.e., $P_{2}$ domains, in comparison to the $\pm P_{1}$ domains which reduce in size. The relaxation process eventually eliminates the misfit stresses of the "1323" domain

TABLE I. The normalized volume average free energy $\tilde{\psi}$, average axial strains $\tilde{\varepsilon}_{i j}$, and polarization $\tilde{P}_{i}$ adopted by rank- 2 periodic domain patterns with in-plane polarization in their settled state.

\begin{tabular}{|c|c|c|c|c|c|}
\hline \multirow{2}{*}{$\begin{array}{l}\text { Rank-2 periodic } \\
\text { domain pattern }\end{array}$} & \multirow{2}{*}{$\begin{array}{c}\text { Average } \\
\text { free energy } \\
\tilde{\psi}\end{array}$} & \multicolumn{2}{|c|}{$\begin{array}{c}\text { Average strain } \\
\text { components } \tilde{\varepsilon}_{i j}=\left\langle\varepsilon_{i j}\right\rangle / \varepsilon_{0}\end{array}$} & \multicolumn{2}{|c|}{$\begin{array}{c}\text { Average polarization } \\
\text { components } \tilde{P}_{i}=\left\langle P_{i}\right\rangle / P_{0}\end{array}$} \\
\hline & & $\tilde{\varepsilon}_{11}$ & $\tilde{\varepsilon}_{22}$ & $\tilde{P}_{1}$ & $\tilde{P}_{2}$ \\
\hline (a) Laminate "1234" & 0.49 & 0.29 & 0.29 & -0.05 & 0.05 \\
\hline (b) Laminate " 1323 " & 0.48 & 0.32 & 0.29 & 0.47 & 0.00 \\
\hline (c) Laminate "1434" & 0.77 & 0.06 & 0.54 & 0.24 & -0.24 \\
\hline (d) Laminate "1324" & 0.75 & 0.28 & 0.28 & 0.00 & 0.00 \\
\hline
\end{tabular}


pattern, resulting in a uniformly polarized stress-free domain at equilibrium.

The domain pattern "1434" shown in Fig. 6(c) contains stripelike and herringbonelike domains. This domain pattern is unstable at the scale simulated $(L=40 \mathrm{~nm})$, dissolving into a monodomain. During relaxation, the $-P_{2}$ domains, which have the largest volume fraction, grow relative to neighboring domains. Referring to Fig. 7, the "1434" pattern has stressed domains which contribute to an increased free energy.

The checkerboard domain pattern " 1324 " contains repeating groups of $90^{\circ}$ domains forming closed polarization loops, or vortices [see Fig. 6(d)]. This pattern too is unstable: it dissolves into a rank-1 stripe domain pattern at equilibrium. As noted by Tsou et al. [18] the checkerboard pattern contains the strongest disclinations among the four in-plane rank-2 domain patterns of Figs. 6(a)-6(d), leading to stresses of order $2 \sigma_{0}$, and hence high energy. This can clearly be observed in the stress distributions of Fig. 7. During relaxation, the domain junctions dissolve by formation of $180^{\circ}$ domain walls. Symmetry breaking occurs and the final state is a rank-1 domain pattern with nonzero average polarization at equilibrium.

Although the herringbone pattern "1234" is well known both at the nano- and microscales, the other patterns shown in Figs. 6(b)-6(d) have relatively rarely been observed and reported [27,18]. A recent work by Tang et al. [28] reports a periodic array of flux-closure domains in $\mathrm{PbTiO}_{3}$. However, this polarization pattern differs from the checkerboard pattern in Fig. 6(d), in that the observed pattern possesses a $180^{\circ}$ domain wall at the vortex core. This domain pattern observed by Tang et al. is referred to in Sec. III C of this paper, where the phase-field simulations indicate its stability in the presence of external loads.

So far, the experimental observations on nanoscale periodic polarization patterns available in literature are in agreement with our phase-field simulations. However, it is of interest to consider how scaling of the periodic cell size affects the energy density of these patterns. Crudely, we can think of the free energy $\int \psi d V$ as including a contribution due to gradient energy $\int \psi_{\mathrm{w}} d V$ and an elastic or dielectric energy part $\int \psi_{\mathrm{e}} d V$. The gradient energy is mainly due to domain walls and scales with domain-wall area. Meanwhile the contributions to the elastic or dielectric energy arise from misfit stress and disclinations, with disclinations being the main factor. For the $i$ th disclination in a given pattern, the energy contribution scales with the square of its disclination angle, $\theta_{i}$, the elastic modulus, $c \sim 67 \mathrm{GPa}$ [78], and the volume $V_{i}$ of material in proximity to the disclination (taken here to be the set of material points closest to the $i$ th disclination). Note the laminates in Figs. 6(b)-6(d) contain disclination dipoles. For example, consider laminate "1324" in Fig. 6(d)_in this pattern, alternating positive and negative disclinations are observed upon circulating domain junctions. The long-range elastic field induced by these disclination dipoles cancel each other and are neglected in the present approximation. A rough estimate of the free energy can then be calculated using

$$
\int \psi(\mathbf{x}) d V \sim \int_{A_{90}} \gamma_{90} d A_{90}+\int_{A_{180}} \gamma_{180} d A_{180}+\sum_{i} c \theta_{i}^{2} V_{i}
$$

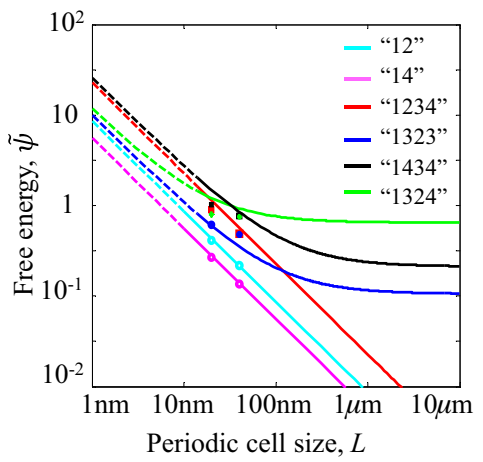

FIG. 8. Estimates of the variation of free energy for rank-1 and rank-2 domain patterns as a function of periodic cell size, $L$. Markers indicate the energy calculated for domain patterns in Figs. 3(a)-3(d) and 6(a)-6(d) using phase-field simulations.

Here $\gamma_{90} \sim 6 \mathrm{~mJ} / \mathrm{m}^{2}$ and $\gamma_{180} \sim 13 \mathrm{~mJ} / \mathrm{m}^{2}$ are the 90 and $180^{\circ}$ domain-wall energies, respectively. $A_{90}$ and $A_{180}$ are the corresponding domain-wall areas within the periodic cell. Dividing through by the periodic cell volume and using the normalization $\tilde{\psi}=\left(\psi_{0}-\psi\right) / \psi_{0}$, the variation of the normalized free energy with scale can be compared for the different patterns (see Fig. 8).

When the periodic cell size is $L<100 \mathrm{~nm}$, the contributions from gradient energy become significant, increasing the energy of each of the rank-1 and rank- 2 domain patterns. Conversely, for periodic cells a few micrometers in size, the elastic energy contribution is dominant, and the energy density approaches a constant for the rank-2 domain patterns with nonzero elastic energy. For the herringbone pattern, "1234" and the rank-1 laminates, the domains are almost stress-free and so the gradient energy is the dominant contribution over a wide range of scale: then $\tilde{\psi} \propto L^{-1}$. The percentage of gradient energy contribution for complex domain patterns such as the checkerboard pattern " 1324 " is low at the microscale, but they have high overall energy because of residual stresses.

The phase-field calculations of free energy, carried out at $L=40$ and $20 \mathrm{~nm}$ for various laminates, are marked in Fig. 8. It was not practically feasible to carry out phase-field simulations over a wide range of scales because of the computational cost, which increases approximately as $L^{4}$. Reducing the periodic cell size much below $L=20 \mathrm{~nm}$ is also problematic because the spacing between domain walls becomes comparable to the domain-wall width-then the domains become unstable. This lower limit of stability is indicated by a transition to dashed lines in Fig. 8 .

Since the area and volume contributions differ from pattern to pattern, some scale dependent cross-over in energies is expected, as seen in Fig. 8. However, the energy estimates are not sufficiently precise to quantify the length scales at which cross-over occurs. In any case, we can see that the simpler, stress-free rank-1 laminates have lower energy than the rank-2 patterns for all sizes of the periodic cell. Stability of the laminates under zero external load conditions does not correspond to a global energy minimum, but rather a local minimum or neutral equilibrium state.

Figure 8 provides a qualitative insight into the domain pattern energies. Rank-2 domain patterns which were unstable 
Initial

(a) "5556"

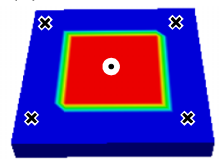

(b) " 5656 "
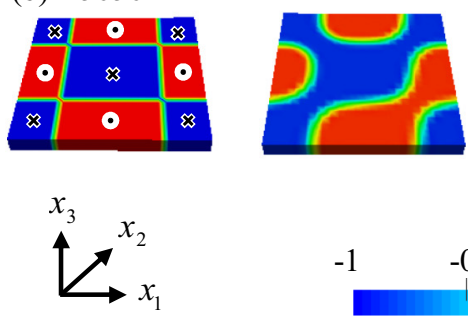

During relaxation
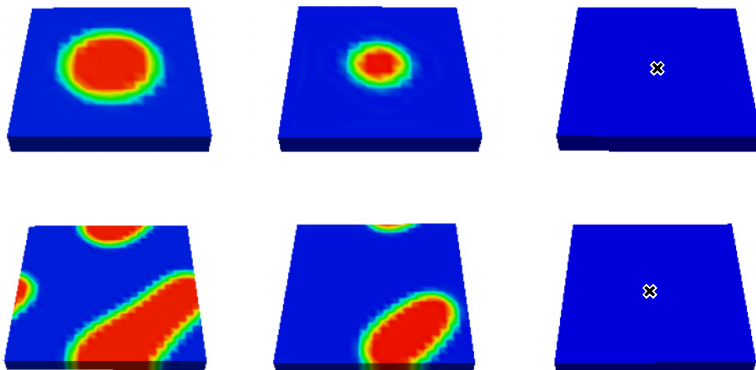

FIG. 9. Testing the stability of rank-2 domain patterns with out-of-plane polarizations $\pm P_{3}$ under zero external load conditions: (a) laminate "5556" and (b) laminate "5656."

at the 40-nm scale under zero external load conditions reduced their gradient energy and elastic energy contributions by evolving into simpler patterns such as rank-1 stripes, or forming a monodomain. By contrast, the herringbone domain pattern "1234" reached equilibrium although having substantial domain-wall energy. Closer examination reveals that this domain pattern is easily dissolved: for example, application of external electric field $E_{2}=-0.05 E_{0} \sim 1 \mathrm{MV} / \mathrm{m}$ dissolves this domain pattern into a monodomain. These findings are important because they suggest that the rank-2 patterns other than the well-known herringbone pattern are relatively unlikely to form at microscale, though they are occasionally seen [18]. This is due to their having high energy from residual stress. However, at the nanoscale the energies of rank- 2 and rank-1 laminates become rather similar. At this scale, the more complex patterns are still energetically unfavorable, but there may be a possibility to stabilize them with stress/strain conditions, or electrical conditions. This opens an exciting possibility of domain engineering at the nanoscale that will be explored further in Sec. III C below.

Next, we test the stability of three rank-2 domain patterns with out-of-plane polarizations under zero external load conditions as described for a 3D model by Eq. (3). In each case we use the minimum periodic cell size that allows the pattern to be represented with sufficient elements to resolve the domain walls. Two of these domain patterns, namely, "5556" and "5656" [see Figs. 9(a) and 9(b)], have a 2D arrangement of domain walls, but out-of-plane polarizations, parallel to $x_{3}$. For these patterns a prismatic platelike periodic cell is used. The third pattern, "1423" (see Fig. 10), has a fully 3D domain-wall arrangement, requiring a thicker periodic cell.

The laminate " 5556 " is initialized with its polarization values on a periodic cell of dimension $16 \times 16 \times 2 \mathrm{~nm}$ [see Fig. 9(a)]. During relaxation, the $180^{\circ}$ domain walls curve to enclose a cylindrical $P_{3}$ domain. The radius of this cylindrical domain reduces to zero, leaving a monodomain at equilibrium. The volume average free energy and average axial components of strain and polarization adopted by this domain pattern in the settled state are given in Table II. It is interesting to note that domain patterns similar to the "5556" laminate, though less regular, are observed experimentally [30,71]. The current phase-field model lacks lattice friction, which is likely to be a factor in stabilizing this domain pattern.

For interest, we test the ability of an external electric field $E_{3}$ to hold a nanoscale cylindrical domain, such as that formed during the relaxation of pattern "5556," in equilibrium. The simulation is initialized with the " 5556 " pattern but subject to the condition

$$
\phi\left(x_{1}, x_{2}, D\right)=\phi\left(x_{1}, x_{2}, 0\right)-E_{3} D .
$$

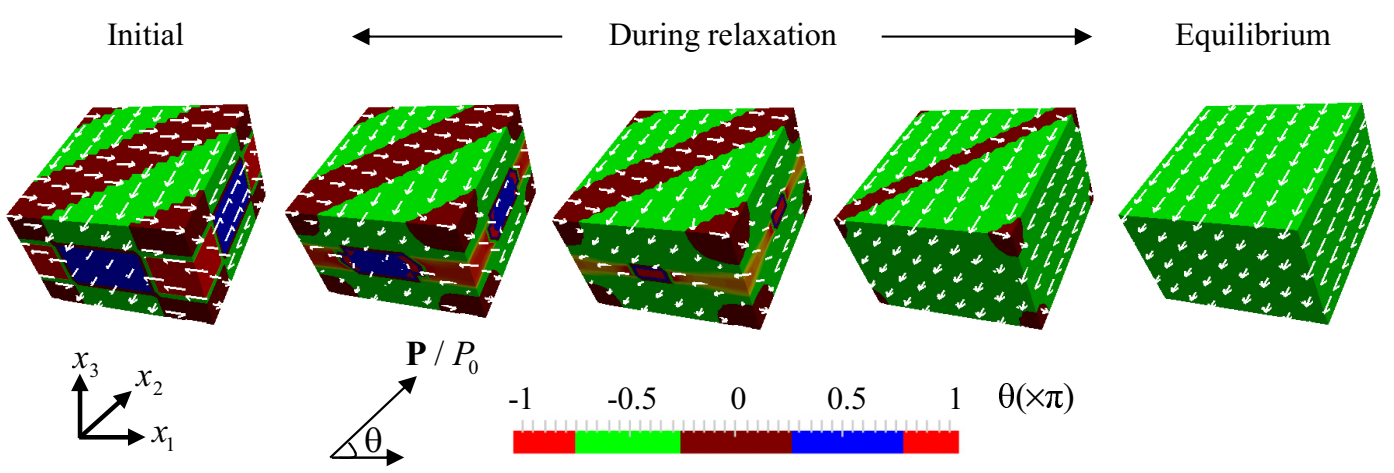

FIG. 10. Testing the stability of rank-2 domain pattern "1423" with 3D domain-wall arrangement under zero external load conditions. 
TABLE II. The normalized volume average free energy $\tilde{\psi}$, average axial strains $\tilde{\varepsilon}_{i j}$, and polarization $\tilde{P}_{i}$ adopted by rank-2 periodic domain patterns with out-of-plane polarization in their settled states.

\begin{tabular}{|c|c|c|c|c|c|}
\hline \multirow{2}{*}{$\begin{array}{l}\text { Rank-2 periodic } \\
\text { domain pattern }\end{array}$} & \multirow{2}{*}{$\begin{array}{c}\text { Average } \\
\text { free energy } \\
\tilde{\psi}\end{array}$} & \multicolumn{2}{|c|}{$\begin{array}{c}\text { Average strain } \\
\text { components } \tilde{\varepsilon}_{i j}=\left\langle\varepsilon_{i j}\right\rangle / \varepsilon_{0}\end{array}$} & \multicolumn{2}{|c|}{$\begin{array}{c}\text { Average polarization } \\
\text { components } \tilde{P}_{i}=\left\langle P_{i}\right\rangle / P_{0}\end{array}$} \\
\hline & & $\overline{\tilde{\varepsilon}_{11}=\tilde{\varepsilon}_{22}}$ & $\tilde{\varepsilon}_{33}$ & $\overline{\tilde{P}_{1}=\tilde{P}_{2}}$ & $\tilde{P}_{3}$ \\
\hline (a) Laminate "5556" & 0.39 & -0.26 & 0.82 & 0.00 & -0.50 \\
\hline (b) Laminate "5656" & 0.70 & -0.27 & 0.86 & 0.00 & 0.00 \\
\hline (c) Laminate " 1423 " & 0.74 & 0.24 & -0.19 & 0.00 & 0.00 \\
\hline
\end{tabular}

Other boundary conditions are as in Eq. (3), giving zero average stress. While it was possible to hold cylindrical domains in equilibrium with electric field, the pattern was unstable to small perturbations in applied field strength, dissolving to a monodomain.

The periodic domain pattern "5656" shown in Fig. 9(b) is imposed on a periodic cell of size $24 \times 24 \times 2 \mathrm{~nm}$, with corresponding polarization values in the initial state. This domain pattern also dissolves into a monodomain. During relaxation, symmetry is broken and the $180^{\circ}$ domain walls curve to form loops, each enclosing a $P_{3}$ domain. This reduces the domain-wall area as discussed by Tagantsev et al. [27]. The eventual collapse to a monodomain is similar to that of the " 5556 " pattern. Patterns with intersecting $180^{\circ}$ domain walls, like "5656," are rarely observed.

The "1423" domain pattern comprises layers of rank1 stripe domain pattern arranged in a three-dimensional configuration as shown in Fig. 10. This domain pattern is initialized on a periodic cell of size $20 \times 20 \times 13 \mathrm{~nm}$. At this periodic cell size, the gradient energy contribution is substantial (see Table II); this destabilizes the domain pattern. However, the layered stripe domain pattern is disclination free. It has been observed in experiments [18,27], and we expect that this domain pattern could reach equilibrium at a larger size of the periodic cell, or with suitable boundary constraints. In other work, the pattern was found stable with a 33-nm cell size, the boundaries of which were constrained to match a fixed strain field [79].

A fourth rank-2 laminate with out-of-plane polarization, "1325," contains domains polarized along all three coordinate axes [18] and requires a minimum periodic cell size of about $60 \mathrm{~nm}$. This pattern is not tested here, due to computational limitations, but is the subject of ongoing work.

To conclude the discussion of rank-2 laminates with zero external load conditions, our key finding is that the majority of these complex laminates are not stable at the nanoscale. The exception is the well-known herringbone domain pattern. The main reasons for the instability of most of the patterns appear to be the high contribution to the overall energy from either disclinations or domain walls. Nevertheless, these structures, if they could be stabilized, offer the possibility of nanoengineering of domain configurations. We thus explore next the possibility of stabilizing these patterns using external loading.

\section{Stabilization by external loading}

Are there loading conditions that can stabilize the rank-2 patterns? To explore this question, we start by considering the average of the spontaneous strain in each of the three laminates "1323," "1434," and "1324," found unstable under zero external load conditions. Suppose a given pattern contains $n$ domains, numbered $k=1 \ldots n$, each having a volume fraction $f_{k}$ and normalized spontaneous strain tensor $\varepsilon_{i j}^{k}$. Neglecting domain-wall volume, the volume average of spontaneous strain in the periodic cell is

$$
\bar{\varepsilon}_{i j}=\sum_{k=1}^{n} f_{k} \varepsilon_{i j}^{k} \quad\left(0 \leqslant f_{k} \leqslant 1, \quad \sum f_{k}=1\right) .
$$

In the simulations of Sec. III B, the periodic cell under zero load conditions given by Eq. (3) was allowed to adopt any value of average strain in order to minimize energy. The resulting equilibrium states have different domain volume fractions from the initial states and hence the average spontaneous strain changes during relaxation. We next conduct simulations in which the average strain is fixed at the $\bar{\varepsilon}_{i j}$ value of the initial state, using the boundary conditions

$$
\begin{aligned}
& \left.u_{1}\left(L, x_{2}\right)\right|_{R}=\left.u_{1}\left(0, x_{2}\right)\right|_{R_{m}}+\bar{\varepsilon}_{11} L, \\
& \left.u_{2}\left(x_{1}, L\right)\right|_{S}=\left.u_{2}\left(x_{1}, 0\right)\right|_{S_{m}}+\bar{\varepsilon}_{22} L
\end{aligned}
$$

in place of the corresponding displacement boundary conditions in Eq. (3). All other boundary conditions remain unchanged. As before, plane simulations were conducted, enforcing $P_{3}=0$ and fixing $\varepsilon_{33}=\varepsilon_{0}^{\mathrm{t}}$. The resulting pattern evolution is shown in Fig. 11: fixing the average strain stabilizes the "1323" laminate, while "1434" and "1324" still collapse into lower-energy patterns. In the case of the "1324" pattern, the $90^{\circ}$ stripe pattern that forms matches the imposed $\bar{\varepsilon}_{i j}$ without stress, while the "1434" collapses to a stressed monodomain.

A further attempt to stabilize the "1434" and "1324" patterns can be made by imposing a fixed average polarization state to match the average spontaneous polarization of the initial state. This discourages the formation of monodomain or simple stripe patterns because they do not match the initial average polarization. Defining $P_{i}^{k}$ as the normalized spontaneous polarization of the $k$ th domain and neglecting domain-wall volume, the volume average polarization in the periodic cell is

$$
\bar{P}_{i}=\sum_{k=1}^{n} f_{k} P_{i}^{k}\left(0 \leqslant f_{k} \leqslant 1, \quad \sum f_{k}=1\right) .
$$

This is applied to the periodic cell by replacing the zero electric-field condition in Eq. (3) with

$$
\left.\phi\left(L, x_{2}\right)\right|_{R}-\left.\phi(L, 0)\right|_{B}=\left.\phi\left(0, x_{2}\right)\right|_{R_{m}}-\left.\phi(0,0)\right|_{A}
$$




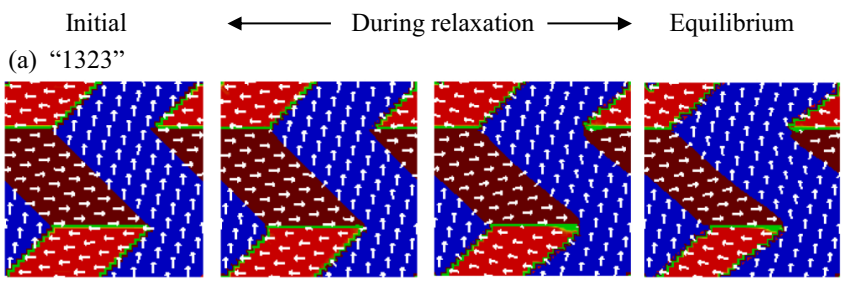

(b) "1434"
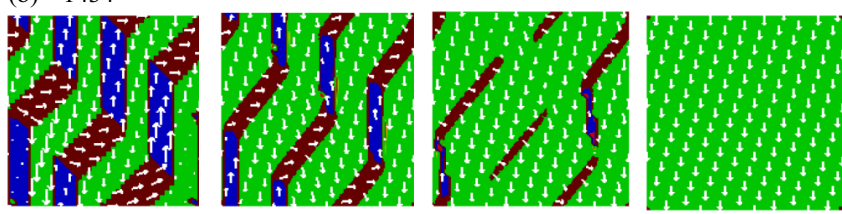

(c) "1324"
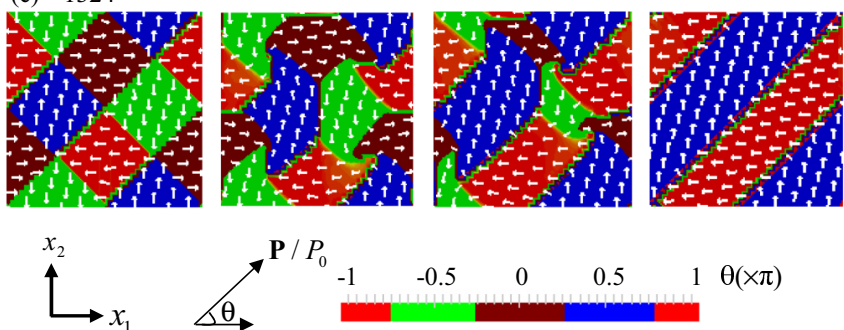

$\underset{\Delta \nabla^{\mathbf{P} / P_{0}}}{\longrightarrow}$

$P_{0}$

$-0.5$

$0 \quad 0.5$

$1 \theta(\times \pi)$

FIG. 11. Evolution of rank-2 laminates (a) "1323," (b) "1434," and (c) "1324," under fixed average strain boundary conditions.

and similar conditions for typical node $S$. These boundary conditions allow an arbitrary, but periodic, electric field. A uniform charge density $q=-\bar{P}_{i} n_{i}$ (where $n_{i}$ is the outward surface normal) can then be added on all boundary nodes to enforce the average polarization. The other boundary conditions are as given in Eq. (3). Note that in the checkerboard pattern "1324" $\bar{P}_{i}=0$. The resulting simulations are shown in Figs. 12(a) and 12(b), where it can be seen that the imposition of an average polarization does stabilize pattern "1434." However, the checkerboard pattern "1324" undergoes some pattern change before stabilizing. During relaxation, $180^{\circ}$ domain walls appear at the $90^{\circ}$ domain junctions, forming a "displaced" checkerboard pattern. Similar features at domain junctions have been imaged in $\mathrm{BaTiO}_{3}$ lamellae by McGilly et al. [15]. The simulations with imposed average strain or polarization demonstrate that periodic laminations of domains can be stabilized by external loading: if domains form in a region of crystal that is mechanically constrained, or has charged surfaces, nanoscale patterns such as these could form. Thus in a prestressed thin film or particle it may be possible to engineer specific nanoscale patterns through the control of the stress/strain and external charge state. This suggests an exciting route towards generating specific arrangements of domains with useful functional properties. However it should be noted that the existence of a stable pattern does not imply that the material will adopt that pattern from arbitrary starting conditions. In the simulations so far, an idealized pattern was imposed as a starting state: this would not normally be possible in a practical device. This leads us to explore whether complex patterns can be generated starting from known simple patterns, and whether they can be transformed or switched by applied fields.

\section{Switching and transformation of patterns}

Can complicated patterns, such as the rank-2 laminates, develop under some conditions, from simpler patterns, such as the rank-1 stripes? To explore this, we study the effect of imposing zero average polarization on rank-1 laminates "14" with $s=14.1$ and $7.1 \mathrm{~nm}$, as shown in Fig. 13. The simulations are initialized with the $90^{\circ}$ stripe domain patterns at equilibrium from Figs. 3(c) and 3(d). Note that these stripe patterns possess a net polarization field and only $90^{\circ}$ domain walls. If we now impose $\bar{P}_{i}=0$, some $180^{\circ}$ domains are expected to form to accommodate the average polarization. In the simulations, during relaxation, the polarization in the domains reduces to near zero magnitude and new domains nucleate such that rank-2 laminate patterns begin to evolve. At first, the strong electric fields generated by imposing a zero average polarization state cause the local polarization vectors to bend far away from the crystallographic axes-a

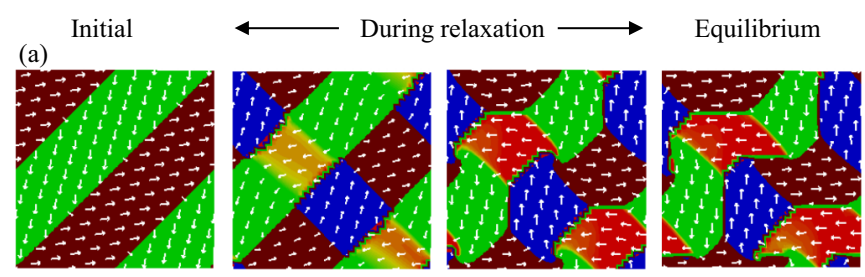

(b)
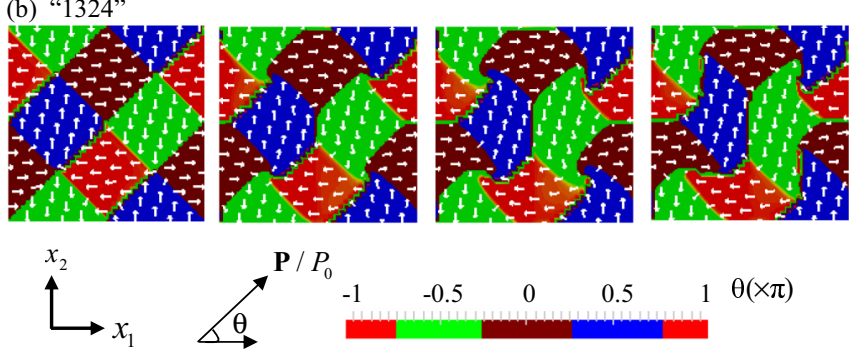

$-0.5 \quad 0$
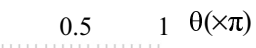

FIG. 12. Evolution of the rank-2 domain patterns (a) "1434" and (b) "1324," under fixed average polarization condition.
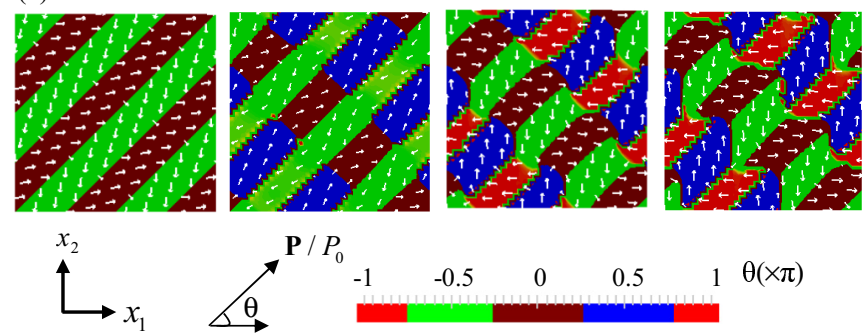

FIG. 13. Evolution of the rank-1 $90^{\circ}$ stripe domain pattern under zero average polarization (a) $s=14.1 \mathrm{~nm}$ and (b) $s=7.1 \mathrm{~nm}$. 
high-energy vortexlike state that must relax further. But as the system approaches equilibrium the polarization in each new domain returns close to the spontaneous value and aligns with the crystal axes. For $s=14.1 \mathrm{~nm}$ [see Fig. 13(a)], a "displaced" checkerboard pattern as seen in Fig. 12(b) is found at equilibrium.

The periodic pattern in Fig. 13(a) is similar to the experimental observation of a periodic array of flux-closure domains in $\mathrm{PbTiO}_{3}$ reported by Tang et al. [28], while with $s=7.1 \mathrm{~nm}$ [see Fig. 13(b)] a herringbone domain pattern is stabilized. Care is needed in interpreting these results: observe that scaling of the cell size in these simulations (or equivalently, changing the domain spacing) leads to different equilibrium states. This happens because the imposed conditions have forced the polarization, at first, far from the spontaneous states, making the subsequent evolution of domains highly sensitive to the kinetics, here represented crudely through the polarization viscosity. Thus the path of domain evolution during switching is to some extent an artifact of the model. Also, from an energetic point of view, the nucleation of the new domains is less costly in the simulation if the pattern adopts the minimum number of periods that will fit into the simulation cell. This explains the way the individual domains form new layers with the minimum possible spatial frequency. This is an artifact of the cell size: in nature there is no imposed cell and the new spacing must arise purely from a balance between the gradient and bulk energies. Nevertheless, the simulations in Fig. 13 indicate the opportunity to engineer complex patterns such as the rank-2 laminates by starting with a simple rank-1 pattern and applying suitable external conditions that force the pattern to nucleate new domains. The length scale of the initial pattern then controls or guides the generation of the new pattern. We have given only a few examples to illustrate the process, but expect that similar methods could enable the generation of a range of patterns.

Finally, we give an example of the effect of electric field on a strain stabilized laminate. Once again, switching is involved, so the cell size and kinetics could affect the simulations. Taking as initial state the stabilized "1323" laminate from Fig. 11(a), additional loading in the form of an electric field of magnitude $0.9 E_{0}=20 \mathrm{MV} / \mathrm{m}$ is applied, and the pattern is relaxed towards a new equilibrium state. If the field is applied along the $\pm x_{1}$ direction the pattern collapses into $90^{\circ}$ stripe domains, by growing whichever domain is aligned to the applied electric field. The electric field in the $+x_{2}$ direction expands the domain polarized in this direction, but the pattern is otherwise unchanged. However, Fig. 14 shows the interesting case where electric field is applied along the $-x_{2}$ direction and there is no domain in the initial state that is aligned with the applied electric field. The pattern undergoes a transition which produces laminate "1424," a symmetry related

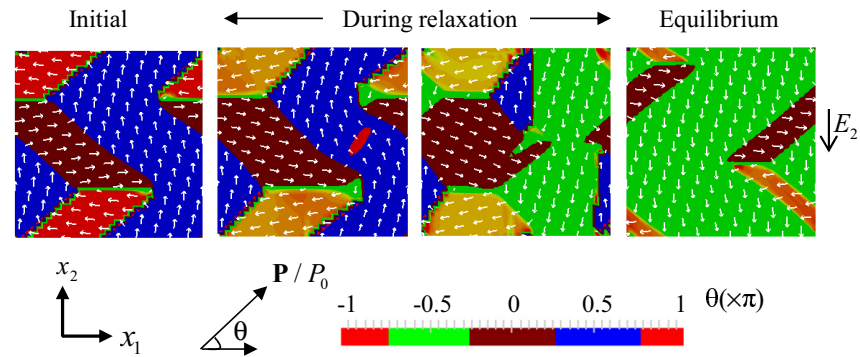

FIG. 14. Evolution of rank-2 domain pattern " 1323 ” stabilized by average strain and under electric field, $E_{2}=-20 \mathrm{MV} / \mathrm{m}$.

pattern in the same family. From symmetry, the process can be reversed by subsequent application of electric field in the $+x_{2}$ direction, suggesting a mechanism for cyclic polarization switching of the nanoscale periodic patterns. Such switchable nanoscale patterns could have great potential for memory elements, or tunable devices.

\section{CONCLUSION}

The stability of rank-1 and rank-2 laminates comprising nanoscale domains formed in tetragonal ferroelectrics was explored using a phase-field model, taking account of energy contributions from gradient energy and elastic strain energy. With zero external load conditions, the rank-1 laminates (simple stripe patterns) were found to be in neutral equilibrium, while the more complex rank-2 laminates were on the whole unstable, with the exception of the herringbone domain pattern. Rank-2 nanoscale domain patterns with multiple domain walls and disclinations were found to possess high-energy density, which destabilized them. The effect of scaling the rank-1 and rank-2 laminates was qualitatively discussed. It was found that the unstable rank-2 domain patterns could be equilibrated under external loads such as electric field, average strain, or polarization, representative residual states of stresses and electric fields that are commonly present or can be imposed on ferroelectric crystals. Finally, the simulations indicated possible routes to generate rank-2 laminated domain patterns from simpler rank-1 domain patterns, and how polarization reversal could occur in nanoscale periodic patterns.

\section{ACKNOWLEDGMENTS}

The authors would like to acknowledge the use of the University of Oxford Advanced Research Computing facility in carrying out this work. A.R.B. acknowledges the support of a scholarship from the Felix Trust. The authors also wish to thank Prof. C. M. Landis for help in providing program codes and advice.
[1] N. Liu, Y. Su, and G. J. Weng, J. Appl. Phys. 113, 204106 (2013).

[2] L. Van Lich, T. Shimada, K. Nagano, Y. Hongjun, J. Wang, K. Huang, and T. Kitamura, Acta Mater. 88, 147 (2015).

[3] Y. W. Li and F. X. Li, J. Appl. Phys. 117, 244101 (2015).
[4] J. Wang, W. Shu, T. Shimada, T. Kitamura, and T. Y. Zhang, Acta Mater. 61, 6037 (2013).

[5] G. Arlt, Ferroelectrics 104:1, 217 (1990).

[6] J. Rödel, Mech. Mater. 39, 302 (2007). 
[7] G. J. Weng and D. T. Wong, J. Mech. Phys. Solids 57, 571 (2009).

[8] J. Y. Li and D. Liu, J. Mech. Phys. Solids 52, 1719 (2004).

[9] L. Liao, H. J. Fan, B. Yan, Z. Zhang, L. L. Chen, B. S. Li, G. Z. Xing, Z. X. Shen, T. Wu, X.W. Sun, J. Wang, and T. Yu, ACS Nano 3, 700 (2009).

[10] X. Feng, B. D. Yang, Y. Liu, Y. Wang, C. Dagdeviren, Z. Liu, A. Carlson, J. Li, Y. Huang, and J. A. Rogers, ACS Nano 5, 3326 (2011).

[11] J. F. Scott, Science 315, 954 (2007).

[12] A. Renuka Balakrishna, J. E. Huber, and C. M. Landis, Smart Mater. Struct. 23, 085016 (2014).

[13] S.-Y. Cheng, N.-J. Ho, and H.-Y. Lu, J. Am. Ceram. Soc. 89, 2177 (2006).

[14] G. Arlt and P. Sasko, J. Appl. Phys. 51, 4956 (1980).

[15] L. J. McGilly, A. Schilling, and J. M. Gregg, Nano Lett. 10, 4200 (2010).

[16] J. M. Ball and R. D. James, Arch. Ration. Mech. Anal. 100, 13 (1987).

[17] K. Bhattacharya, Contin. Mech. Thermodyn. 5, 205 (1993).

[18] N. T. Tsou, P. R. Potnis, and J. E. Huber, Phys. Rev. B 83, 184120 (2011).

[19] A. DeSimone and R. D. James, J. Mech. Phys. Solids 50, 283 (2002).

[20] C. M. Dudhe, S. B. Nagdeote, S. J. Khambadkar, P. R. Arjunwadkar, and R. R. Patil, Ferroelectrics 471, 148 (2014).

[21] J. F. Scott, J. Phys. Condens. Matter 18, R361 (2006).

[22] O. Kolosov, A. Gruverman, J. Hatano, K. Takahashi, and H. Tokumoto, Phys. Rev. Lett. 74, 4309 (1995).

[23] V. Gopalan, V. Dierolf, and D. A. Scrymgeour, Annu. Rev. Mater. Res. 37, 449 (2007).

[24] H. H. Wu, J. Wang, S. G. Cao, L. Q. Chen, and T. Y. Zhang, Smart Mater. Struct. 23, 025004 (2014).

[25] Y. H. Hu, H. M. Chan, Z. X. Wen, and M. P. Harmer, J. Am. Ceram. Soc. 69, 594 (1986).

[26] C. T. Nelson, P. Gao, J. R. Jokisaari, C. Heikes, C. Adamo, A. Melville, S.-H. Baek, C. M. Folkman, B. Winchester, Y. Gu, Y. Liu, K. Zhang, E. Wang, J. Li, L.-Q. Chen, C.-B. Eom, D. G. Schlom, and X. Pan, Science 334, 968 (2011).

[27] A. K. Tagantsev, L. E. Cross, and J. Fousek, Domains in Ferroic Crystals and Thin Films (Springer, New York, 2010).

[28] Y. L. Tang, Y. L. Zhu, X. L. Ma, A. Y. Borisevich, A. N. Morozovska, E. A. Eliseev, W. Y. Wang, Y. J. Wang, Y. B. Xu, Z. D. Zhang, and S. J. Pennycook, Science 348, 547 (2015).

[29] A. Schilling, R. M. Bowman, G. Catalan, J. F. Scott, and J. M. Gregg, Nano Lett. 7, 3787 (2007).

[30] J. A. Hooton and W. J. Merz, Phys. Rev. 98, 409 (1955).

[31] F. Xue, X. S. Gao, and J.-M. Liu, J. Appl. Phys. 106, 114103 (2009).

[32] L. Jin, Z. Xi, Z. Xu, and X. Yao, Ceram. Int. 30, 1695 (2004).

[33] A. Renuka Balakrishna and J. E. Huber, Appl. Phys. Lett. 106, 092906 (2015).

[34] J. M. Gregg, Ferroelectrics 433, 74 (2012).

[35] G. Catalan, J. Seidel, R. Ramesh, and J. F. Scott, Rev. Mod. Phys. 84, 119 (2012).

[36] E. K. H. Salje, Chem. Phys. Chem 11, 940 (2010).

[37] R. Ahluwalia and W. Cao, Phys. Rev. B 63, 012103 (2000).

[38] J. Wang, S.-Q. Shi, L.-Q. Chen, Y. Li, and T.-Y. Zhang, Acta Mater. 52, 749 (2004).

[39] W. Cao, S. Tavener, and S. Xie, J. Appl. Phys. 86, 5739 (1999).
[40] S. Nambu and D. A. Sagala, Phys. Rev. B 50, 5838 (1994).

[41] G. J. Weng, Acta Mech. 225, 979 (2014).

[42] A. Kontsos and C. M. Landis, Int. J. Solids Struct. 46, 1491 (2009).

[43] A. Kontsos and C. M. Landis, J. Appl. Mech. 77, 041014 (2010).

[44] L.-Q. Chen, J. Am. Ceram. Soc. 91, 1835 (2008).

[45] L.-Q. Chen, Annu. Rev. Mater. Res. 32, 113 (2002).

[46] H. T. Chen, S. D. Zhang, A. K. Soh, and W. Y. Yin, J. Appl. Phys. 118, 034106 (2015).

[47] L. Jiang, Y. Zhou, Y. Zhang, Q. Yang, Y. Gu, and L.-Q. Chen, Acta Mater. 90, 344 (2015).

[48] G. Catalan, A. Lubk, A. H. G. Vlooswijk, E. Snoeck, C. Magen, A. Janssens, G. Rispens, G. Rijnders, D. H. A. Blank, and B. Noheda, Nat. Mater. 10, 963 (2011).

[49] A. Y. Borisevich, E. A. Eliseev, A. N. Morozovska, C.-J. Cheng, J.-Y. Lin, Y. H. Chu, D. Kan, I. Takeuchi, V. Nagarajan, and S. V. Kalinin, Nat. Commun. 3, 775 (2012).

[50] R. Ahluwalia, A. K. Tagantsev, P. Yudin, N. Setter, N. Ng, and D. J. Srolovitz, Phys. Rev. B 89, 174105 (2014).

[51] Y. Gu, M. Li, A. N. Morozovska, Y. Wang, E. A. Eliseev, V. Gopalan, and L.-Q. Chen, Phys. Rev. B 89, 174111 (2014).

[52] E. A. Eliseev, A. N. Morozovska, Y. Gu, A. Y. Borisevich, L. Q. Chen, V. Gopalan, and S. V. Kalinin, Phys. Rev. B 86, 085416 (2012).

[53] P. V. Yudin, A. K. Tagantsev, E. A. Eliseev, A. N. Morozovska, and N. Setter, Phys. Rev. B 86, 134102 (2012).

[54] E. A. Eliseev, P. V. Yudin, S. V. Kalinin, N. Setter, A. K. Tagantsev, and A. N. Morozovska, Phys. Rev. B 87, 05411 (2013).

[55] W. Zhang and K. Bhattacharya, Acta Mater. 53, 185 (2005).

[56] J. J. Wang, X. Q. Ma, Q. Li, J. Britson, and L. Q. Chen, Acta Mater. 61, 7591 (2013).

[57] I. Steinbach, Model. Simul. Mater. Sci. Eng. 17, 073001 (2009).

[58] J. Li, C. Lei, L. Li, Y. Shu, and Y. Liu, Acta Mech. Sin. 28, 915 (2012).

[59] S. Choudhury, Y. Li, C. Krill III, and L. Chen, Acta Mater. 53, 5313 (2005).

[60] J. Wang, Y. Li, L. Chen, and T. Zhang, Acta Mater. 53, 2495 (2005).

[61] S. Choudhury, Y. L. Li, C. Krill III, and L. Q. Chen, Acta Mater. 55, 1415 (2007).

[62] A. K. Soh, Y. C. Song, and Y. Ni, J. Am. Ceram. Soc. 89, 652 (2006).

[63] Y. Cao, G. Sheng, J. X. Zhang, S. Choudhury, Y. L. Li, C. A. L. Randall, and L. Q. Chen, Appl. Phys. Lett. 97, 252904 (2010).

[64] F. Xue, J. J. Wang, G. Sheng, E. Huang, Y. Cao, H. H. Huang, P. Munroe, R. Mahjoub, Y. L. Li, V. Nagarajan, and L. Q. Chen, Acta Mater. 61, 2909 (2013).

[65] H.-L. Hu and L.-Q. Chen, J. Am. Ceram. Soc. 81, 492 (1998).

[66] N. T. Tsou, J. E. Huber, and A. C. F. Cocks, Acta Mater. 61, 670 (2013).

[67] Y. Su and C. M. Landis, J. Mech. Phys. Solids 55, 280 (2007).

[68] G. Goldsztein, J. Mech. Phys. Solids 49, 899 (2001).

[69] P. Forsbergh, Phys. Rev. 76, 1187 (1949).

[70] A. Schilling, S. Prosandeev, R. G. P. McQuaid, L. Bellaiche, J. F. Scott, and J. M. Gregg, Phys. Rev. B 84, 064110 (2011).

[71] V. Grubsky, S. MacCormack, and J. Feinberg, Opt. Lett. 21, 6 (1996). 
[72] W. Cao and L. E. Cross, Phys. Rev. B 44, 5 (1991).

[73] J. Hlinka and P. Márton, Phys. Rev. B 74, 104104 (2006).

[74] K. Shapovalov, P. V. Yudin, A. K. Tagantsev, E. A. Eliseev, A. N. Morozovska, and N. Setter, Phys. Rev. Lett. 113, 207601 (2014).

[75] E. A. Eliseev, A. N. Morozovska, S. V. Kalinin, Y. Li, J. Shen, M. D. Glinchuk, L. Q. Chen, and V. Gopalan, J. Appl. Phys. 106, 084102 (2009).
[76] J. Padilla, W. Zhong, and D. Vanderbilt, Phys. Rev. B 53, R5969(R) (1996).

[77] V. Anbusathaiah, D. Kan, F. C. Kartawidjaja, R. Mahjoub, M. A. L. Arredondo, S. Wicks, I. Takeuchi, J. Wang, and V. Nagarajan, Adv. Mater. 21, 3497 (2009).

[78] B. L. Cheng, M. Gabbay, W. Duffy, and G. Fantozzi, J. Mater. Sci. 31, 4951 (1996)

[79] I. Muench, A. Renuka Balakrishna, and J. E. Huber (unpublished). 